\title{
Searching for impossible objects: Processing form and attributes in early vision
}

\author{
NICK DONNELLY \\ University of Kent, Canterbury, England \\ and \\ ANDREW FOUND and HERMANN J. MÜLLER \\ Birkbeck College, University of London, London, England
}

\begin{abstract}
In five experiments, we investigated the extent to which form (shape) and metric attributes (three-dimensional, 3-D, orientation), both defined by relations between line elements, are processed in early vision. Search for a target defined by an abstract property of form (i.e., impossibility) was slow and serial. In contrast, search for a 3-D orientation target was considerably easier. Subsequent experiments suggest that this difference reflects the fact that 3-D orientation is derivable from localized sets of lines, whereas impossibility is an idiosyncratic property of the complete set of relations between lines. We conclude that only "gross" aspects of form are available in early vision as the complete set of line relations is not processed preattentively. However, localized processing of line relations is sufficient to derive 3-D orientation.
\end{abstract}

One of the most important functions of the visual system is to recognize objects within our immediate visual environment. To achieve this, the visual system processes a great deal of information concerning, for example, brightness, color, and motion. However, given such a complex representation of the visual world, it is interesting to note that object recognition itself appears largely insensitive to much of this information and is primarily based on contour information (see Biederman \& Ju, 1988; Ostergaard $\&$ Davidoff, 1985). Although the processing of color and motion may facilitate the derivation of contour (line) information, line information alone appears to provide the input to object recognition processes (see Davidoff, 1991, for a review of evidence). An understanding of how line relations are processed is therefore central to an understanding of object recognition.

In computer vision, a number of algorithms have been developed to interpret line relations and generate object descriptions (see Ballard \& Brown, 1982). Most begin by assigning interpretations of lines and junctions and then proceed by eliminating possibilities based on assumptions of "global coherence." Global coherence means that assignments are discarded as incorrect if they result in a line between two vertices changing interpretation. Such algorithms are reasonably successful within limited "blocks

This work was supported by a grant from the Economic and Social Research Council of Great Britain awarded to N.D. and H.J.M. The authors thank Jan Theeuwes, Jeremy Wolfe, Jim Enns, and an anonymous reviewer for their incisive and constructive criticisms of earlier drafts. Correspondence should be addressed to N. Donnelly, Department of Psychology, University of Kent, Canterbury, Kent CT2 7LZ, U.K. (e-mail: n.donnelly@ukc.ac.uk).

Accepted by previous editor, Myron L. Braunstein world" domains, which allow a relatively restricted set of line and junction labels. However, as the domain of such algorithms increases, the number of possible junction labels increases dramatically (e.g., Waltz, 1975).

The development of such algorithms has been guided by correlations between their output and the interpretations of human observers (with unrestricted viewing). However, it seems unlikely that humans and machines use the same form of line-labeling algorithms. One example of why this is so is seen in the relative difficulty humans have in detecting impossibility in specially constructed "objects." Impossible objects consist of line drawings that depict shapes that could not be "manufactured" in three dimensions. When line-labeling algorithms are presented with impossible objects (such as those in Figure 1), in contrast to humans, machines readily detect the impossibility. These issues have been investigated experimentally by Cowie and Perrott (1993), who instructed participants to view shapes similar to those depicted in Figure 2 (the "devil's torpedo tube"). Participants were instructed to decide whether or not the two dots were on the same object. They were shown this impossible figure only after a sequence of normal (possible) drawings and were under no time pressure to respond. Seventy-five percent of the participants reported that the dots were on the same object. This suggested that participants were generating a possible version of the impossible figure on which they were basing their decisions. In a further experiment, Cowie and Perrott showed that "same" responses were as fast to the impossible figure as they were to the possible figure, which suggests that the erroneous possible representation of the impossible figure is derived as rapidly as its possible counterpart.

Some authors have suggested that humans engage in a restricted form of line-labeling. Enns and Rensink (1991) proposed the PRISM model of processing line relations 

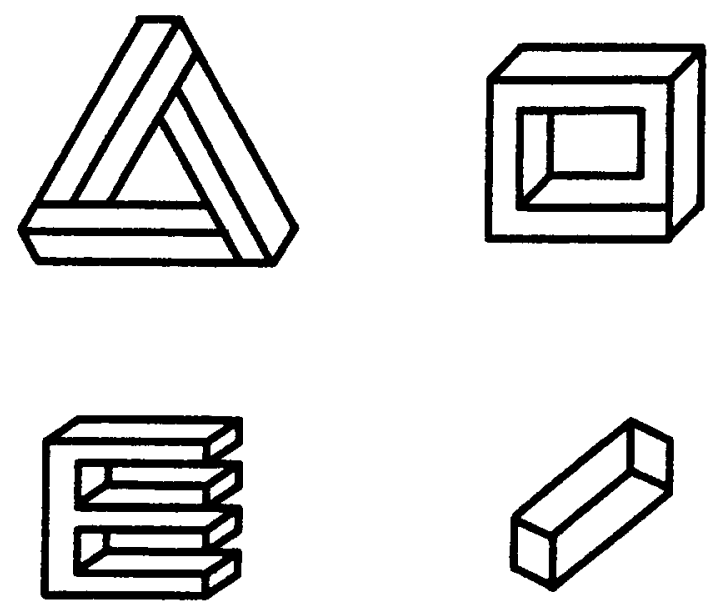

Figure 1. Examples of figures where human observers find it difficult to detect the impossibility.

in early (i.e., preattentive) vision. They suggested that line relations are processed in two stages (the stages operate sequentially, but processing within each stage is parallel). First, $\mathrm{T}$ junctions and $\mathrm{Y}$ junctions are processed simultaneously to derive occlusion and orientation estimates, respectively. The parallel nature of this stage means that properties such as three-dimensional (3-D) orientation are processed in parallel in preattentive vision (Enns \& Rensink, 1991). A second stage of consistency checking takes place where pairwise comparisons of neighboring local estimates are made. The difficulties observers had in Cowie and Perrott's experiment are consistent with Enns and Rensink's idea that initial line labeling and/or consistency checking is noisy and error prone.

The two-stage system suggested by Enns and Rensink has a similar structure to other models that distinguish between object identity (i.e., form) and metric attributes (e.g., orientation and size) (Treisman \& Gelade, 1980; Wolfe, 1994). In these models, processing in early vision is concerned with making estimates of a variety of attributes that objects may or may not possess (color, size, etc.). In contrast, attentive visual processing deals with objects (i.e., their identity, etc.). Thus, a target that differs from distractors in terms of orientation is easy to detect, since search can be performed on the basis of the representation of the metric attribute of orientation that is derived in early vision. However, a task that required participants to search for aspects of shape would be slow and serial, requiring attentive processing.

This distinction between form and attributes makes intuitive sense; however, it becomes somewhat blurred in Enns and Rensink's (1991) model. The 3-D orientation targets used by Enns and Rensink were not distinguishable from distractors on the basis of possessing some unique "primitive" metric attribute. The target was not a different color or size, and it was composed of the same line elements as the distractor items. Rather, the target item differed in terms of the arrangement of these line elements.
The target line elements were arranged in such a way that it was the same shape as the distractors but possessed a different 3-D orientation. Therefore, for Enns and Rensink, the derivation of orientation is at least partially dependent on the computation of shape information.

In the present paper, we report a series of experiments in which we investigated the rate at which impossible objects are detected and the mechanisms that are used to detect them. Using the speed of orientation judgments as a baseline measure, we examined whether impossibility is signaled by a preattentive or attentive process. We were interested in whether impossible objects might be signaled in preattentive vision by an incoherence detection mechanism.

\section{GENERAL METHOD}

\section{Participants}

Six participants took part in Experiments 1, 3, and 5; 8 participants took part in Experiments 2 and 4 . All participants had normal or corrected vision.

\section{Apparatus}

All stimuli were generated by a Silicon Graphics Indigo UNIX workstation. Stimuli were presented on a high-performance color CRT. Responses were recorded through a four-button response box (only the leftmost and rightmost buttons were used).

\section{Stimuli}

Stimulus details varied for each experiment and so are described separately for each experiment.

\section{Procedure}

Each block of trials began with a red cross presented in the center of a black screen $80 \mathrm{~cm}$ away. The participants initiated a block by pressing a button on a mouse situated next to them. Each trial began with a small fixation cross presented in the middle of the screen for $1 \mathrm{sec}$, which was then replaced by the experimental display. The participants were allowed $5 \mathrm{sec}$ to respond. Feedback was presented after every response in the form of a large gray plus $(+)$ sign (a correct response) or minus (-) sign (incorrect response).

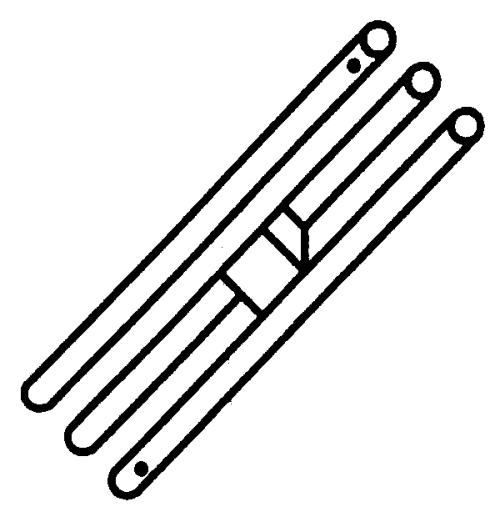

Figure 2. The "devil's torpedo tubes" used by Cowie and Perrott (1993) to investigate the perception of impossible objects. The participants were instructed to decide whether the two dots were on the same object or not. The participants showed a strong tendency to respond that the dots were on the same object, even though they are not. 
The plus sign remained on the screen for $1 \mathrm{sec}$, the minus sign remained on the screen for $2 \mathrm{sec}$ to discourage errors. After feedback, the screen blanked for $1 \mathrm{sec}$ before the next trial began or the end of a block was reached. If the participants failed to respond within $5 \mathrm{sec}$ of display onset, the feedback for an incorrect response was presented and the trial was discarded. Before starting each condition, the participants were given $20-30$ practice trials to ensure that they understood and felt comfortable with the task.

\section{EXPERIMENT 1}

Enns and Rensink (1991) have proposed that a certain property of line relations (i.e., 3-D orientation) is available in early vision. In Experiment 1, we contrasted search for 3-D orientation of a shape with search for the impossible version of a shape. Experiment 1 consisted of two conditions (depicted in Figure 3), the impossible condition and the orientation condition, in which the participants searched for either an impossible target or an oriented target item, respectively. In this experiment, we compared the relative efficiency with which participants searched for an impossible target and a 3-D orientation target.

\section{Method}

Participants and Apparatus. The participants and apparatus were as described in the General Method section. The only additional information is that each condition consisted of 270 trials, 45 present trials and 45 absent trials at each of three set sizes. Trials were presented in a random order in blocks of 45 trials.

Stimuli. Displays in the three conditions consisted of one, four, or seven items arranged radially about a fixation cross. Example target-present displays for the two conditions are shown in Figure 3. Each display item subtended approximately $1.2^{\circ}$ horizontally and $1.4^{\circ}$ vertically.

Procedure. The procedure was as described in the General Method section. In addition, note that the participants were not instructed to search for the impossible/oriented figure but were simply shown the target item and told to respond on the basis of its presence/absence.

\section{Results}

Mean reaction times (RTs; excluding those greater than 4,000 msec, less than $1 \%$ of trials), percent error rates, and standard deviations from mean RTs were subjected to 2 (condition: impossible and orientation) $\times 2$ (response: present and absent) $\times 3$ (set size: 2,4 , and 7 ) analyses of variance (ANOVAs). The analysis of mean RTs demonstrated that all three main effects were significant [condition, $F(1,5)=47.06, p<.01$; response, $F(1,5)=34.67$, $p<.01$; set size, $F(2,10)=217.84, p<.0001$; see Figure 4]. Condition interacted with response $[F(1,5)=36.03$, $p<.01]$ and set size $[F(2,10)=67.81, p<.001]$. The three-way interaction between condition, response, and set size was also significant $[F(2,10)=26.44, p<.001]$. The cause of these interactions is clear from the results of linear regressions. In the orientation condition, the present and absent slope values were 29.6 and $27.3 \mathrm{msec} / \mathrm{item}$, respectively. In the impossible condition, the slope values were 110.9 and $187.6 \mathrm{msec} /$ item for "present" and "absent" responses, respectively. An analysis of the error rates revealed an identical pattern of effects, which reinforced the RT differences between the two conditions.

Analysis of the standard deviations revealed an important interaction between condition, response, and set size $[F(2,10)=12.04, p<.01]$. The three-way interaction was caused by increased variability with set size on impossible present trials relative to impossible absent trials. There was no difference in the rate of increased variability with set size for present and absent trials on the orientation task.

\section{Discussion}

The difference between the two conditions is clear: Search for an orientation difference, generated by a structural rearrangement of line segments, was much more efficient than was search for an incoherence in the line re-

\section{Impossible Condition}

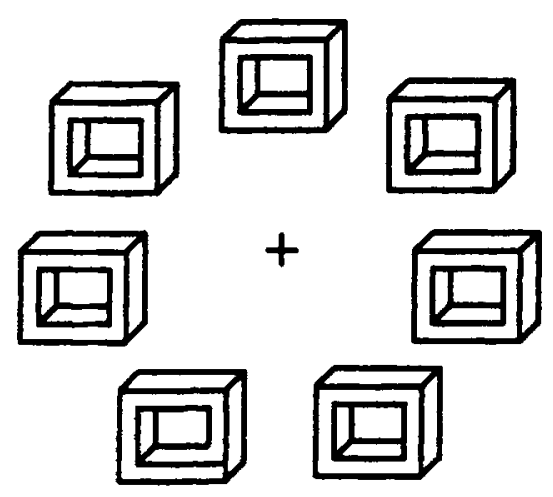

\section{Orientation Condition}

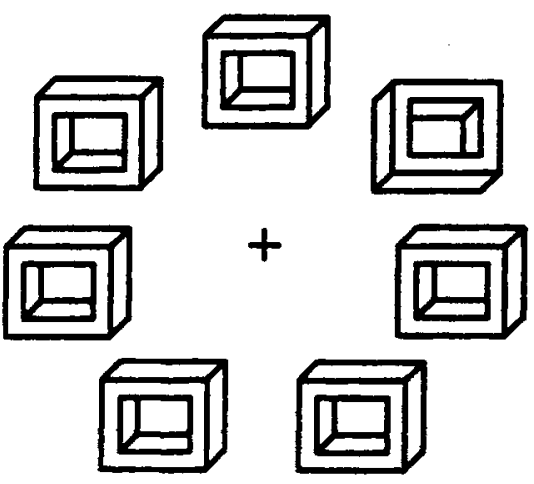

Figure 3. Example displays in the two conditions of Experiment 1 (both contain 7 items). In the impossible condition (left), the target is an impossible object (bottom left); in the orientation condition, the target is oriented differently to distractors (top right). 


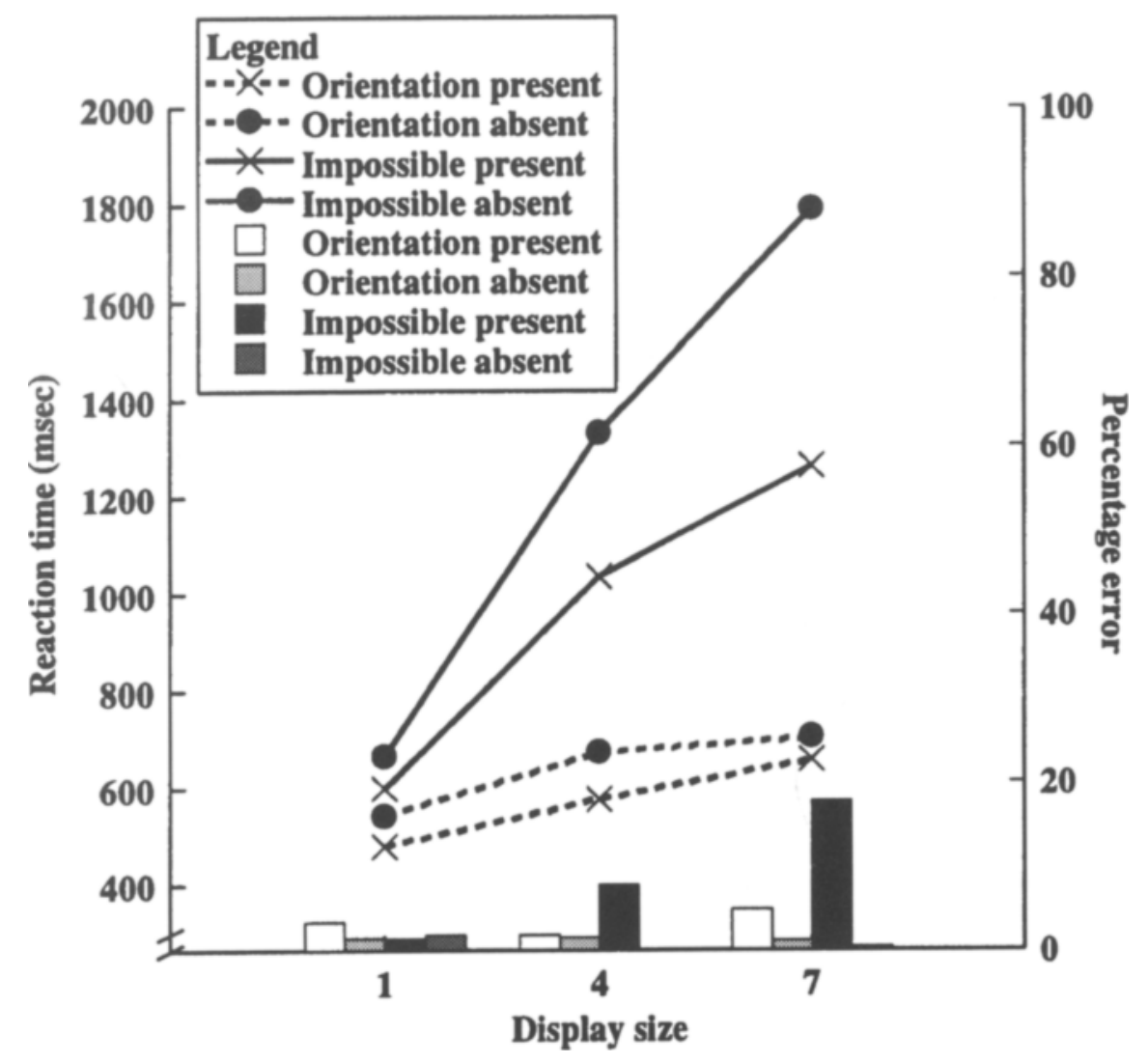

Figure 4. Mean RTs and error rates for "present" and "absent" responses in the impossible and orientation conditions of Experiment 1.

lations themselves. Not only was search more efficient in the orientation condition than in the impossible condition, the ratio of present:absent slopes suggests that search might have been qualitatively different in the two conditions. In the orientation condition, the present and absent slope ratio was virtually $1: 1(1: 0.9)$, whereas, in the impossible condition, the ratio was much higher (1:1.7). Traditionally, such slope differences have been used to argue for parallel and serial searches, respectively. However, previous theoretical treatments (e.g., Townsend, 1972) and recent empirical analyses (see Wolfe, 1997) suggest that we should be more circumspect in determining the nature of visual search solely from the relative slopes of "absent" and "present" responses. This is especially so given that computing confidence intervals (see Loftus \& Masson, 1994) for the orientation task (mean $\pm 48 \mathrm{msec}$ ) reveals that, although unlikely, a 1:2 present:absent slope could be found within the $95 \%$ limits surrounding mean RTs. In the present case, the concern is heightened as mean slope for the orientation task was $28.5 \mathrm{msec} / \mathrm{item}$, and some researchers have used a cutoff of $\approx 10 \mathrm{msec} / \mathrm{item}$ to differentiate between parallel and serial searches. Using this criterion, both of the conditions of Experiment 1 would be thought of as being performed in serial, with differences in the size of the slope being determined by such factors as stimulus quality. Poor stimulus quality might lead to eye movements being necessary before a stimulus can be categorized as target or distractor. Given the size of the slopes, the most likely case for this is in the impossible target detection task. However, a control study in which participants were presented with either an impossible or a possible version of the box figure demonstrated that stimulus quality was not a real concern. In this control study, stimuli were presented individually for $192 \mathrm{msec}$ (too short to allow eye movements to be made), and 6 naive participants were asked to make a possible/impossible decision across a block of 180 trials (plus minimal practice, in line with that presented in Experiment 1 ). The error rates were $13 \%$ and $12 \%$ for "absent" and "present" responses, respectively. These results show that, even for the impossibility detection task, let alone the orientation detection task, the relatively steep slopes found cannot be caused by poor stimulus quality necessitating eye movements.

We need some other criterion that can be used to support the argument that orientation judgments are performed in a qualitatively different fashion from impossibility judgments. Some support can be found in the analyses of standard deviations from mean RTs. Serial self-terminating searches should lead to a greater increase in variation on 
present trials than on absent trials (see Donnelly, Weekes, Humphreys \& Albon, 1998; Treisman \& Gelade, 1980, Experiment 1). In Experiment 1, standard deviations increased more on present trials than on absent trials in the impossible detection task, but they increased equally in the orientation task. Therefore, from the criteria of both RT and standard deviation slope differences (though not absolute slopes themselves), orientation targets are detected from a limited-capacity parallel search, but impossible objects require a serial self-terminating search. Although the usual arguments about having sufficient statistical power to reject the null hypothesis of a parallel search still apply (see Wolfe, 1997), the weight of evidence from two measures (mean RTs and their standard deviations) supports a distinction between making orientation and impossibility judgments. The distinction we draw can certainly be made on quantitative grounds (in terms of relative slopes) but may also be qualitative - that is, a limitedcapacity parallel process (for orientation) versus a serial and self-terminating process (for impossibility).

These data suggest an interesting dissociation between the form of objects and their associated metric attributes (i.e., size and orientation). This dissociation may reflect the fact that identity requires that the entire set of line relations be processed, whereas 3-D orientation can be reliably derived from local sets of line elements associated with particular vertices (see Enns \& Rensink, 1991). We explored this issue in Experiment 2 in which participants were instructed to search for the 3-D orientation of impossible shapes. In particular, we were interested in determining whether the inability to compute a possible object would influence the detection of object orientation.

\section{EXPERIMENT 2}

It was reasoned that, if 3-D orientation was a local property of vertices as opposed to complete objects, then participants should be able to detect the orientation of impossible objects, even though the complete set of line relations should not be represented consistently. We explored this in Experiment 2 using the same "box" stimuli employed in Experiment 1.

Three conditions were tested. In all three conditions, the target item was a box oriented vertically and to the right among distractors oriented downward and to the left. In the possible condition, all display items were possible shapes. In the impossible condition, all display items were impossible shapes. In the mixed condition, the possibility/ impossibility of the target and distractors varied randomly with respect to one another (all distractors on a given trial were either possible or impossible, and any target item was either possible or impossible).

\section{Method}

Participants and Apparatus. The participants and apparatus were as described in the General Method section.

Stimuli. Displays in the three conditions are shown in Figure 5. Other details were as described in the General Method section.

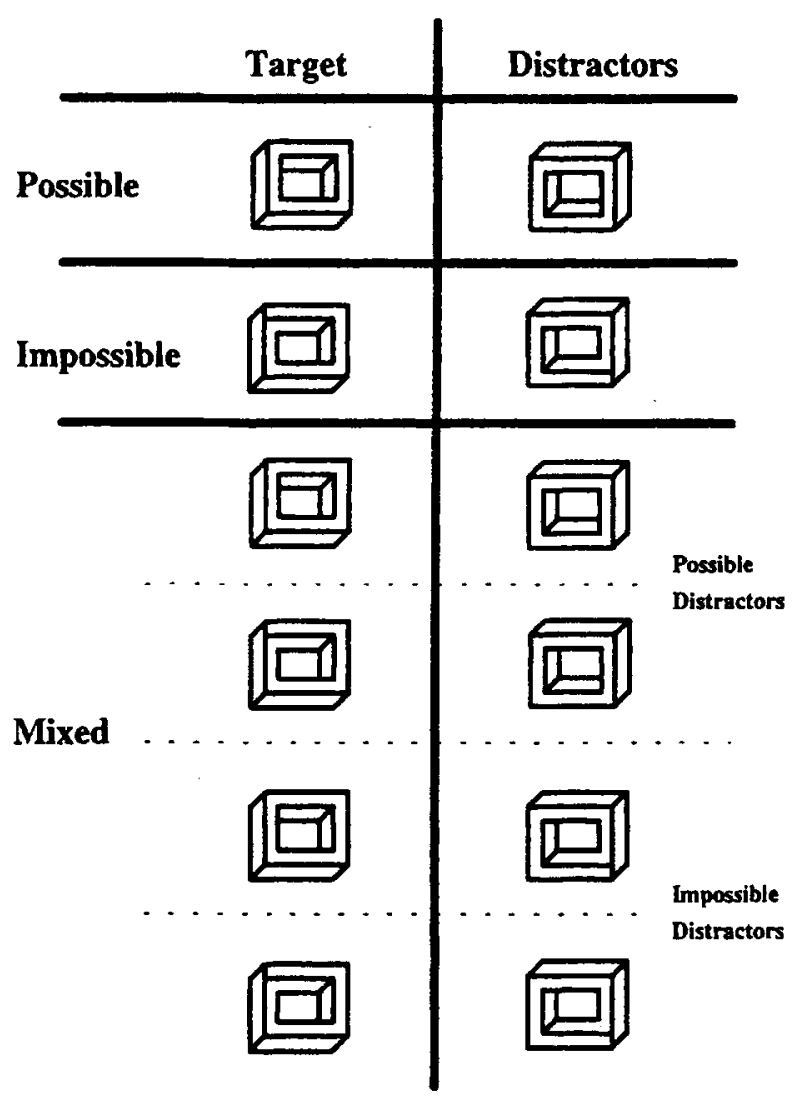

Figure 5. Target and distractor pairs used in the three conditions of Experiment 2 (the mixed condition consisted of four potential target and distractor pairs, which were equally likely).

Procedure. The procedure was as described in the General Method section, except for the following details: The possible and impossible conditions consisted of 270 trials. The mixed condition consisted of 264 trials, with the participants performing two "runs."

\section{Results}

The results were analyzed as in Experiment 1 except that RTs greater than 2,500 msec (less than $1 \%$ of trials) were removed. For RTs, the main effects of condition and response were just significant and the main effect of set size was highly significant [condition, $F(2,14)=3.87, p<$ .05 ; response, $F(1,7)=5.91, p<.05$; set size, $F(2,14)=$ $26.86, p<.0001$; see Figure 6]. The interaction between condition and set size was significant $[F(4,28)=4.23, p<$ $.01]$; however, the interaction between response and set size was not $[F(2,14)=1.19, p=.335]$. For the errors, the main effect of condition was also significant $[F(2,14)=$ $6.60, p<.01]$. In addition, there were no significant effects involving response and set size in the analysis of standard deviations.

ANOVAs comparing mean RTs of conditions in pairs revealed that only differences between the possible and impossible conditions approached significance $[F(1,7)=$ $4.79, .075>p<.05]$. However, ANOVAs comparing er- 

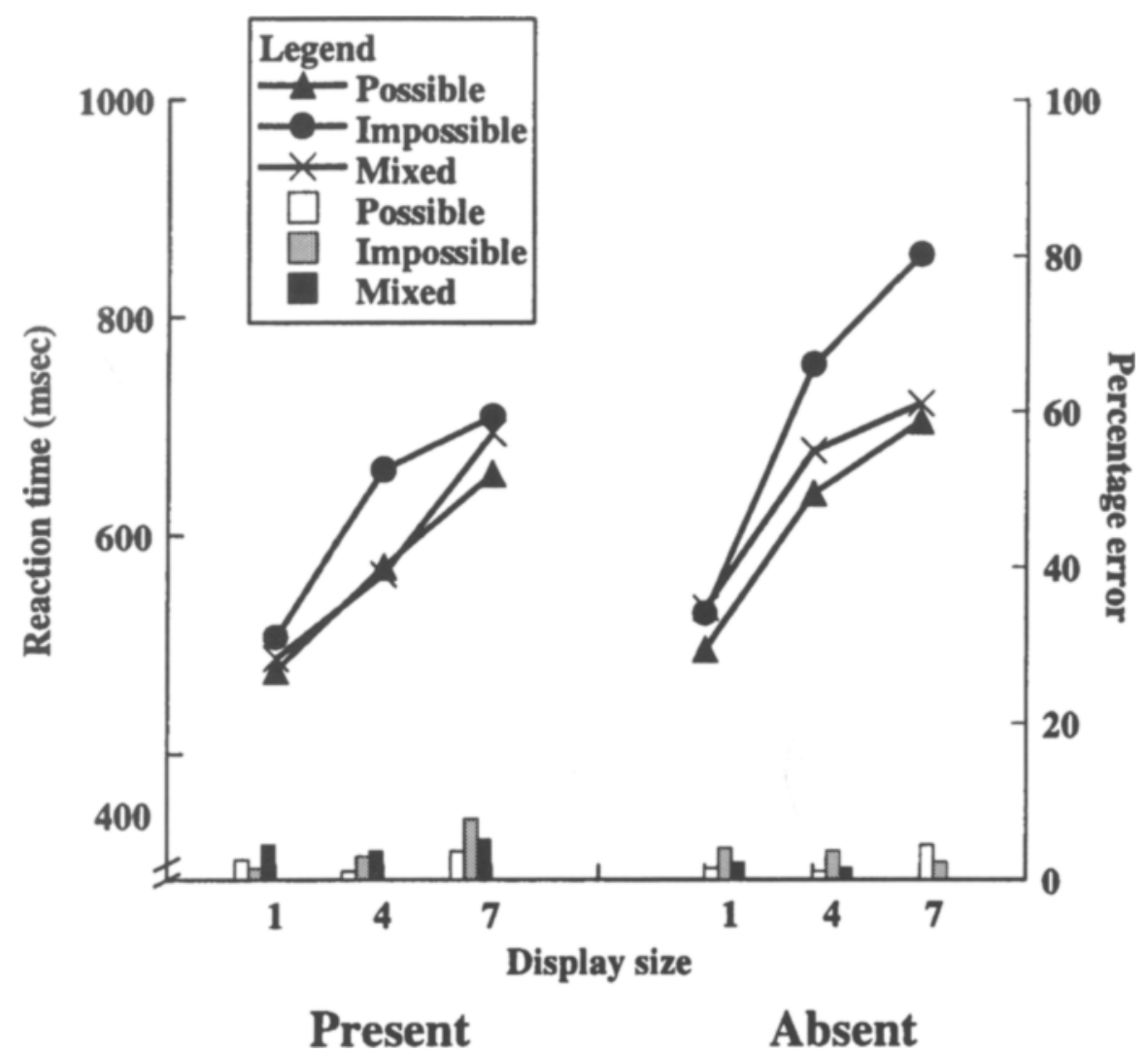

Absent

Figure 6. Mean RTs and error rates for both "present" and "absent" responses for the three conditions of Experiment 2.

rors revealed that the possible condition differed significantly from the impossible condition $[F(1,7)=9.32, p<$ $.05]$ and the mixed condition $[F(1,7)=34.65, p<.01]$. The impossible and mixed conditions did not differ significantly from each other $[F(1,7)=0.61]$. Overall, this pattern of results is consistent with the participants' finding search in the possible condition somewhat easier than in the impossible and mixed conditions. The results of linear regressions performed on present and absent RTs in the three conditions are consistent with this interpretation. Present slopes for the possible, impossible, and mixed conditions were 31,36 , and $35 \mathrm{msec} /$ item, respectively (absent slopes were 31,50 , and $39 \mathrm{msec} /$ item, respectively).

Figure 7 shows mean RTs and errors in the mixed condition according to whether distractors were possible or impossible for possible and impossible targets and for target-absent trials. RTs were faster for displays containing possible distractors. RTs were also faster for possible targets, with both possible and impossible distractors.

An ANOVA of the present trials in the mixed condition, with factors of distractor (possible and impossible), target (possible and impossible), and set size, revealed that only the main effects of target $[F(1,7)=12.31, p<.01]$ and set size $[F(2,14)=26.72, p<.0001]$ were significant. Present RTs were significantly faster to possible orientation targets irrespective of whether distractors were possible or impossible. No interactions were significant. Present RTs for possible targets were approximately $50 \mathrm{msec}$ faster than RTs to impossible targets. A similar analysis comparing absent trials revealed that mean absent RTs to impossible distractors were significantly slower than RTs to possible distractors $[F(1,7)=18.67, p<.01]$, and this difference increased with set size [distractor $X$ set size interaction, $F(2,14)=11.90, p<.01]$. Errors on absent trials showed a similar pattern of effects.

\section{Discussion}

Target-present slopes in the possible and impossible conditions were comparable ( 31 and $36 \mathrm{msec} / \mathrm{item}$, respectively), suggesting that the participants did not find it difficult to search for the 3-D orientation of an impossible object. However, target-absent slopes were somewhat different between these two conditions ( 31 and $50 \mathrm{msec} / \mathrm{item}$, respectively). This suggests that the participants were less confident of the targets' absence in the impossible condition, and they performed a certain amount of "rechecking." Presumably, the participants' difficulty in forming a "stable percept" in the impossible condition was responsible for this uncertainty. This difference between possible and impossible display items was also ev- 


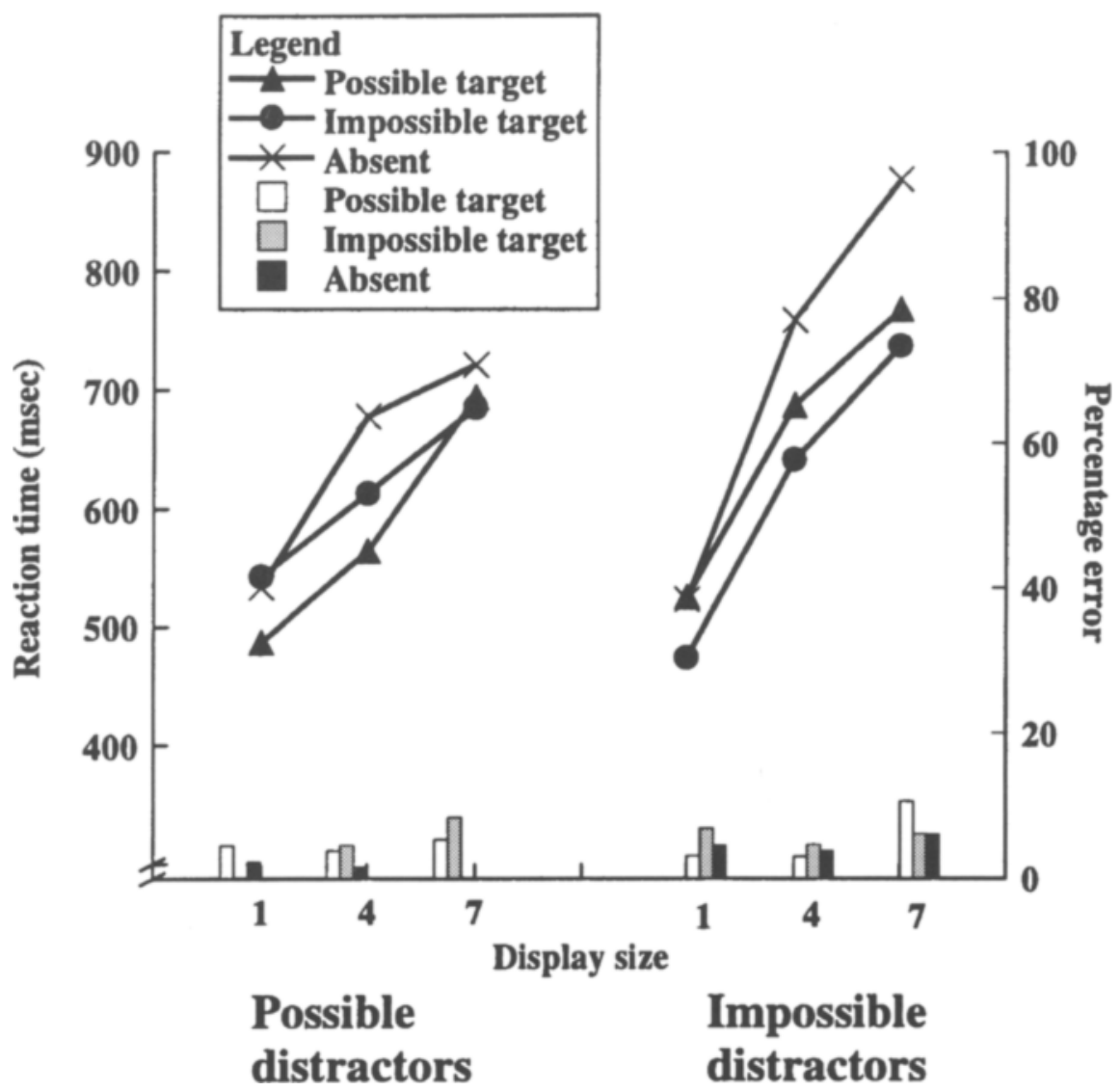

Figure 7. Mean RTs and error rates for responses in the mixed condition of Experiment 2 for both possible and impossible distractors, for displays containing possible and impossible targets, and for target-absent trials.

ident in the results of the mixed condition. Absent RTs were significantly longer to impossible distractors in both possible and impossible target conditions. Interestingly, irrespective of the identity of the distractors, present RTs were significantly faster to possible targets. The absence of an interaction between set size and target identity (possible vs. impossible) suggests that the participants did not have difficulty locating the impossible target but instead were less confident in their responses. Again, presumably due to the fact that they were more readily able to generate a stable percept of the possible target items.

Importantly, comparing across Experiments 1 and 2, the rate of detection for orientation differences with the impossible figures was not of the same magnitude as that for detecting the impossibility itself. Experiments 1 and 2

Table 1

Reaction Times (in Milliseconds), Along With Intercepts, Slopes, and $r^{2}$ Values, for the Control Experiments Reported Under Experiment 2

\begin{tabular}{|c|c|c|c|c|c|c|c|c|c|c|c|c|}
\hline \multirow[b]{3}{*}{ Target/Distractor } & \multicolumn{6}{|c|}{ Target Present } & \multicolumn{6}{|c|}{ Target Absent } \\
\hline & \multicolumn{3}{|c|}{ Display Size } & \multirow[b]{2}{*}{ Intercept } & \multirow[b]{2}{*}{ Slope } & \multirow[b]{2}{*}{$r^{2}$} & \multicolumn{3}{|c|}{ Display Size } & \multirow[b]{2}{*}{ Intercept } & \multirow[b]{2}{*}{ Slope } & \multirow[b]{2}{*}{$r^{2}$} \\
\hline & 1 & 4 & 7 & & & & 1 & 4 & 7 & & & \\
\hline 回回 & 473 & 587 & 653 & 451 & 30 & .98 & 501 & 610 & 712 & 467 & 35 & 1.00 \\
\hline 烏四 & 461 & 568 & 617 & 448 & 26 & .96 & 470 & 594 & 661 & 445 & 32 & .97 \\
\hline 回回 & 483 & 589 & 632 & 469 & 25 & .94 & 488 & 634 & 739 & 453 & 42 & .99 \\
\hline 回回 & 520 & 636 & 726 & 490 & 34 & .99 & 470 & 595 & 661 & 448 & 32 & .97 \\
\hline$\square$ & 501 & 678 & 776 & 468 & 46 & .97 & 520 & 798 & 1,071 & 429 & 92 & 1.00 \\
\hline 氤回 & 530 & 778 & 965 & 468 & 72 & .99 & 553 & 969 & 1,261 & 456 & 118 & .99 \\
\hline
\end{tabular}




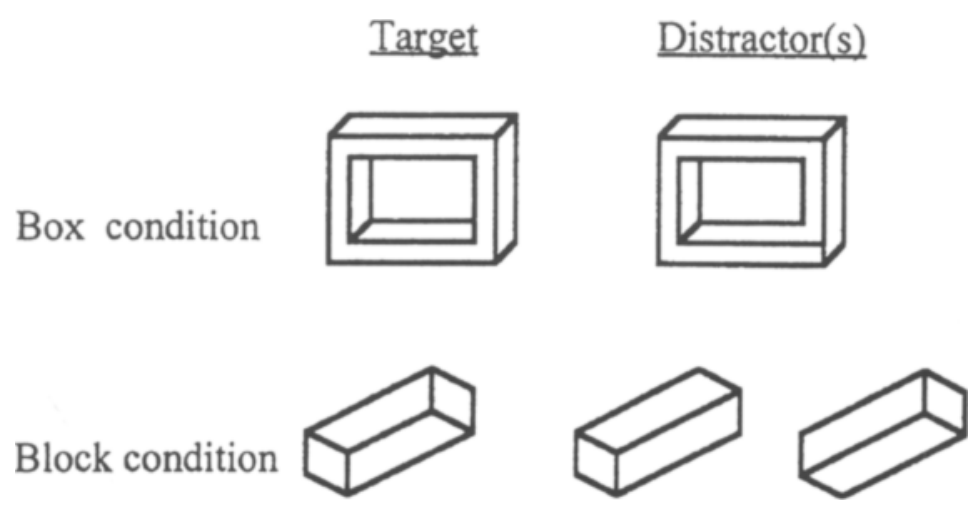

Figure 8. Target and distractor pairs shown in Experiment 3.

have demonstrated that orientation is searched for much more efficiently than is impossibility. However, there is a modest cost associated with detecting the orientation of an impossible object that is consistent with object form influencing decisions about orientation because of inconsistencies that arise during checking. Nevertheless, the results indicate that orientation is available before form information is computed.
There are two alternative explanations as to why impossible objects are more difficult to detect than oriented objects. First, it might be that the spatial resolution required for making orientation judgments is less than that required for impossibility judgments. However, the control study reported in the Discussion section of Experiment 1 ruled out spatial resolution as a key difference between up/down orientation and impossible/possible

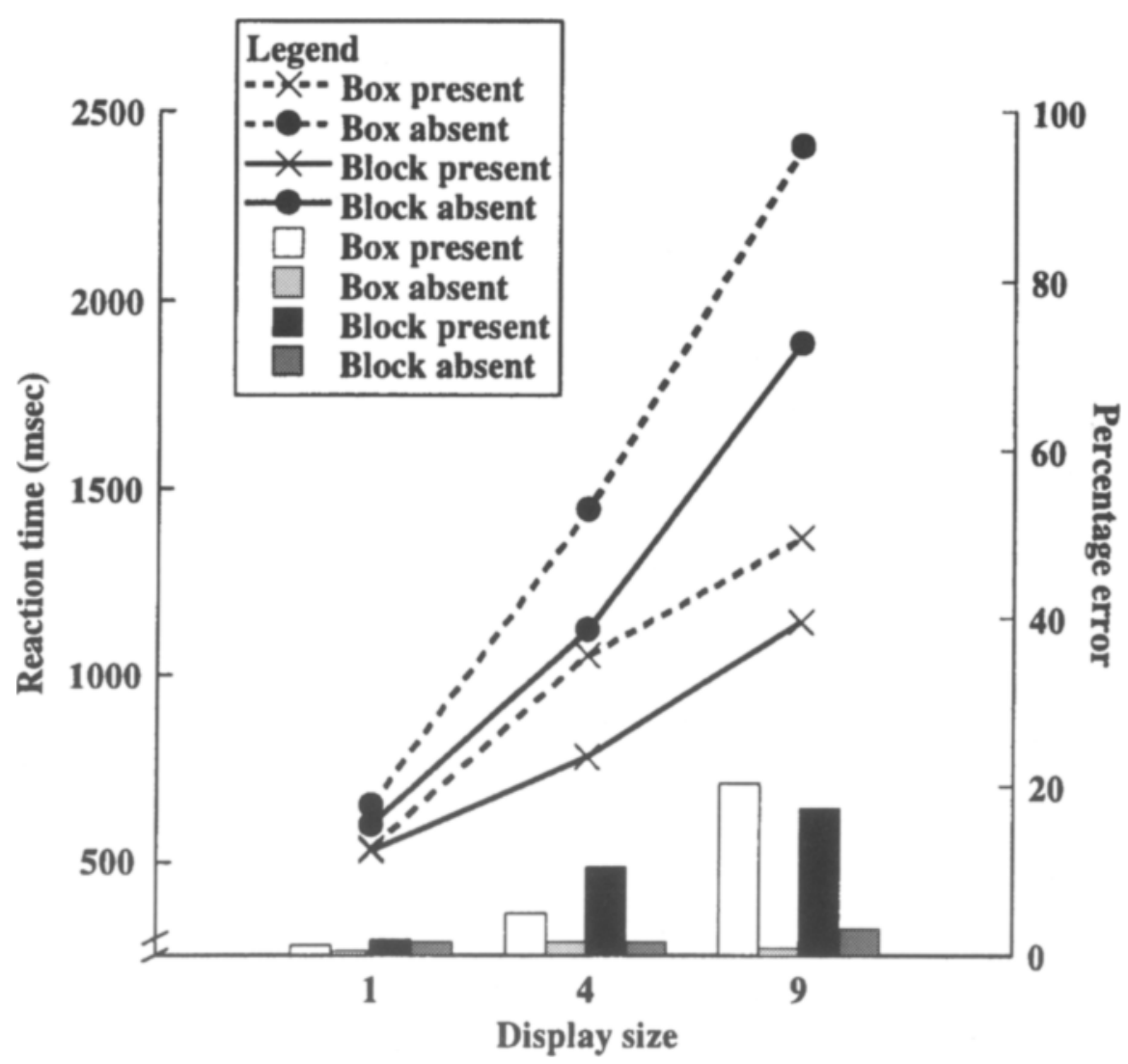

Figure 9. Mean RTs and error rates for present and absent conditions of Experiment 3. 
discriminations. Second, it might be that decisions about orientation can be made from information that can be extracted more readily than that required for impossibility judgments. The information required to make the possible/ impossible decision with the box stimulus is clear (the presence of a $\mathrm{T}$ junction connecting on the sole internal upright); however, it is less obvious what information is critical when making orientation judgments. In a series of control experiments, we attempted to discover the basic information that could be presented maintaining the box form while still allowing the efficient determination of orientation. The results (presented in Table 1) show that orientation judgments are not affected by the removal of inner or outer $Y$ junctions but are slowed when front and back box faces appear disconnected or are not orthogonal (as happens when the outer $\uparrow$ junctions are removed). These results on disconnection are reminiscent of the results of the experiments by Humphreys and Donnelly (1998; see also Humphreys, Keulers, \& Donnelly, 1994, Experiment 1), who examined search across a single 3-D object. The results concerning the importance of orthogonality are similar to those of Enns and Rensink (1991). In short, these control experiments indicated that orientation may be signaled by a pair of $\uparrow$ junctions on the external shape boundary. Therefore, this control study indicates that orientation and impossibility decisions differ in terms of the number, location, and type of key features that are used to reach decisions.

The box figures used in Experiments 1 and 2 have little resemblance to those figures shown by Enns and Rensink (1991) to allow the parallel computation of orientation. Indeed, the differences in stimulus construction between the simple block figures of Enns and Rensink and the box figures shown in Experiments 1 and 2 might account for the relative steepness of the slopes found in our experiments relative to those reported by Enns and Rensink. Although we believe that our data on orientation show qualitatively similar effects to those of Enns and Rensink, in Experiment 3, we investigated the perception of another impossible figure, but one that was more similar to the figure used by them. Our first task was to investigate whether the impossibility present in this figure required a serial self-terminating search, like that required by the box figure shown in Experiments 1 and 2.

\section{EXPERIMENT 3}

The "block" figure is formed from a conjunction of $Y$ vertices along with two end cuts (see Figure 8). Given the angular relations between the lines at vertices, the ends of the block are mutually incompatible (Sugihara, 1982); consequently, the block has no dominant orientation. A possible block figure with a dominant orientation can be created by using only one irregular end cut. Importantly, possible and impossible figures do not vary in the position of critical form information. We compared this new block figures with the box figure shown in Experiments 1 and 2 .

\section{Method}

Participants and Apparatus. The participants and apparatus were as described in the General Method section.

Stimuli. Displays in the two conditions consisted of one, four, or nine items. Displays in the box condition were as in Experiments 1 and 2. Displays in the block conditions contained a heterogeneous set of two different distractors (see Figure 8). In this condition, when set size and target presence/absence allowed, the number of the two distractors was equal; otherwise, the identity of the final distractor item was determined randomly. When displays contained one item, the item was presented at the center of the display area. When set size was four or nine items, these were arranged in a grid pattern again centered on the middle of the display area. When set size was four, the vertical and horizontal separation between the centers of display items was $2.2^{\circ}$. When the display contained nine items, this separation was reduced somewhat to $1.7^{\circ}$. The actual position of items was varied randomly by between $-0.1^{\circ}$ and $+0.1^{\circ}$ both horizontally and vertically. Therefore, when set sizes were four and nine, the entire display subtended approximately $3.45^{\circ} \times 3.6^{\circ}$ and $4.65^{\circ} \times 4.8^{\circ}$, respectively. All other details were as described in the General Method section.

Procedure. The participants were allowed 8 sec to respond. All other details were as in the General Method section. The participants' task was to search for the presence or the absence of an impossible object.

\section{Results}

The results were analyzed as in Experiment 1, but with the condition factor replaced by stimulus type (block and box). Mean RTs, percent error rates, and standard deviation from mean RTs were calculated excluding responses over 4,000 msec (less than $3 \%$ of trials).

For RTs, all main effects were significant [condition, $F(1,5)=26.11, p<.001 ;$ response, $F(1,5)=90.44, p<$

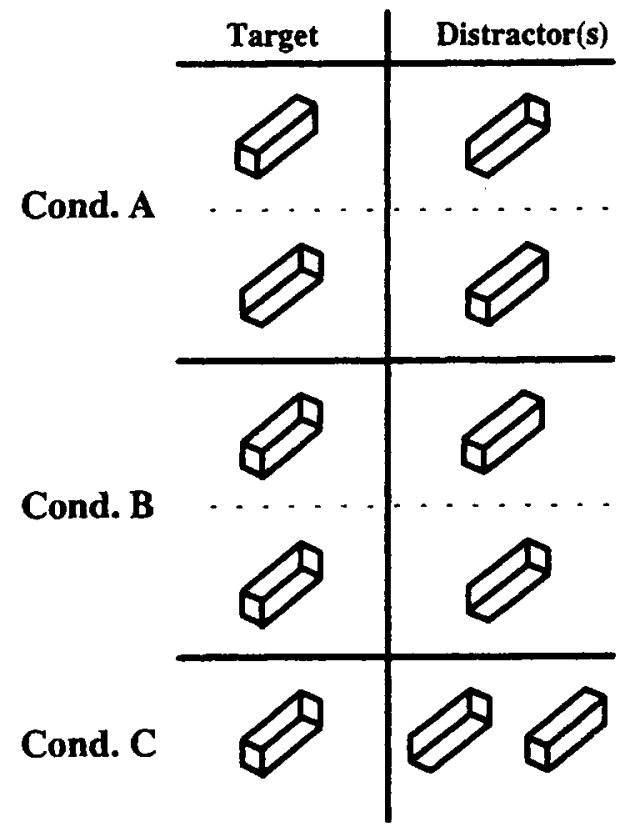

Figure 10. Target and distractor pairings used in the three conditions of Experiment 4. 


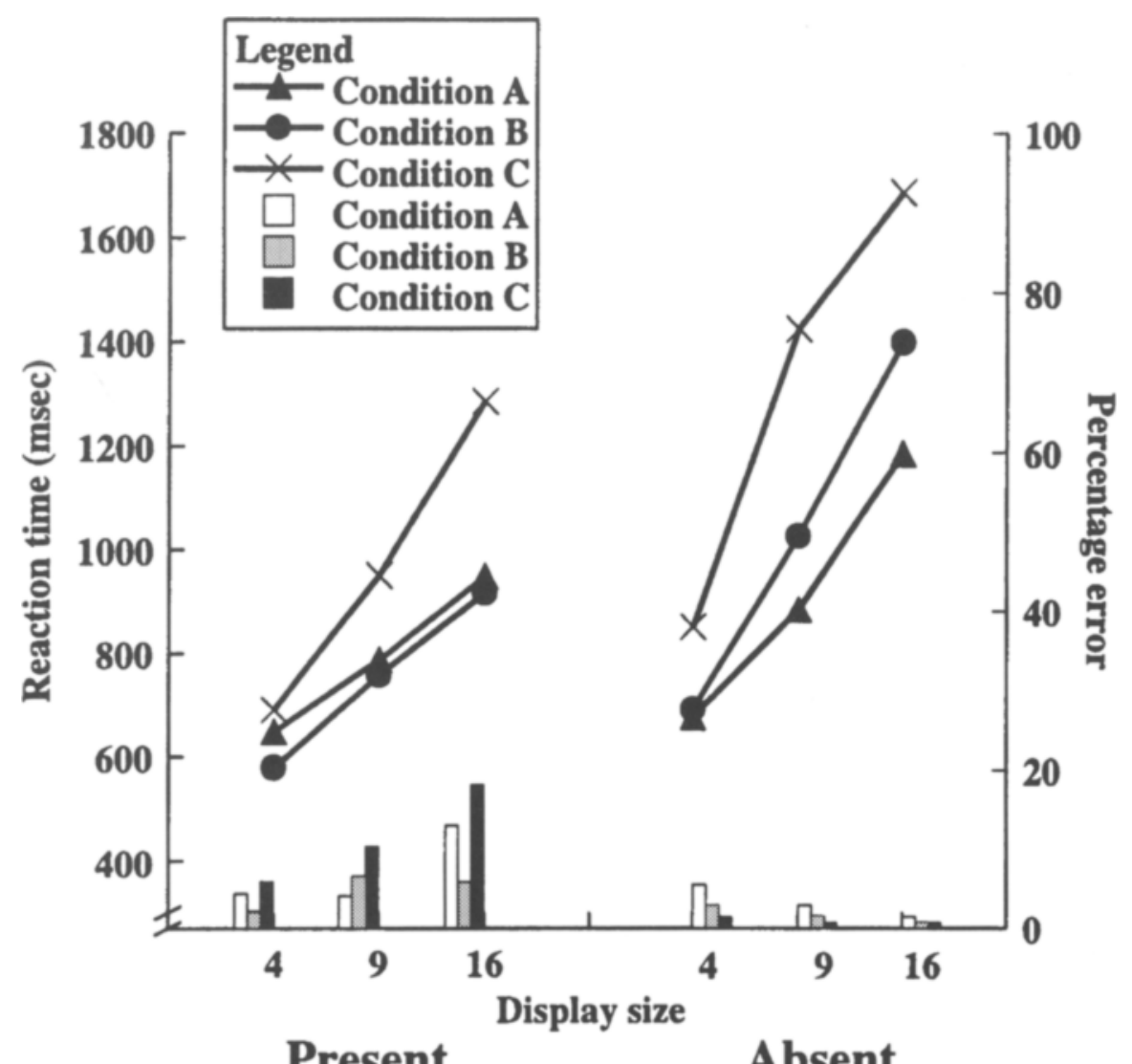

Figure 11. Mean RTs and error rates for both "present" and "absent" responses in the three conditions of Experiment 4.

.001 ; set size, $F(2,10)=108.6, p<.001$; see Figure 9]. RTs were significantly different between the two stimulus types, RTs increased significantly as set size increased, and absent RTs were significantly longer than present RTs. In addition, all of the two-way interactions were significant, with absent RTs increasing more than present RTs as set size increased $[F(2,10)=38.58, p<.001]$. The stimuli differed with respect to the effect of set size on RTs $[F(2,10)=14.44, p<.001]$ and with respect to differences between "present" and "absent" responses $[F(2,10)=$ $23.24, p<.01]$.

The three-way interaction was significant $[F(2,10)=$ $6.02, p<.05]$. Absent/present slope differences were greater with the box figure than with the block figure. This is underlined by the results of linear regressions performed on the mean RTs. Present slopes were 76 and $100 \mathrm{msec} /$ item for the block and box figures, respectively, and for absent slopes, 160 and $217 \mathrm{msec} / \mathrm{item}$, respectively.

A similar analysis of the errors revealed the main effects of response $[F(1,5)=37.05, p<.001]$ and set size $[F(2,10)=14.93, p<.001]$ to be significant. The participants' errors increased as set size increased, and they made many more errors on present trials (misses). The interaction between response and set size was also significant $[F(2,10)=16.93, p<.001]$.

A similar analysis of standard deviations revealed an important significant interaction between stimulus type, response, and set size $[F(2,10)=28.93, p<.01]$. Standard deviations increased with display size in all conditions but they increased twice as much on present trials with the box figure as they did with the block figure. There were no other significant differences between conditions.

\section{Discussion}

The results of Experiment 3 are clear, and they replicate those of Experiment 1: The participants found searching for both of the impossible objects difficult. In addition, the present:absent slope ratios in all conditions were close to $1: 2$, indicative of serial self-terminating search. It is worthwhile to note here that a control experiment, identical to that reported in the Discussion section of Experiment 1 but using the block figure, confirmed that the impossible/possible discrimination could be performed easily without eye movements $(6 \%$ and $5 \%$ errors on present and absent trials, respectively, for the same group of 6 participants reported earlier). 

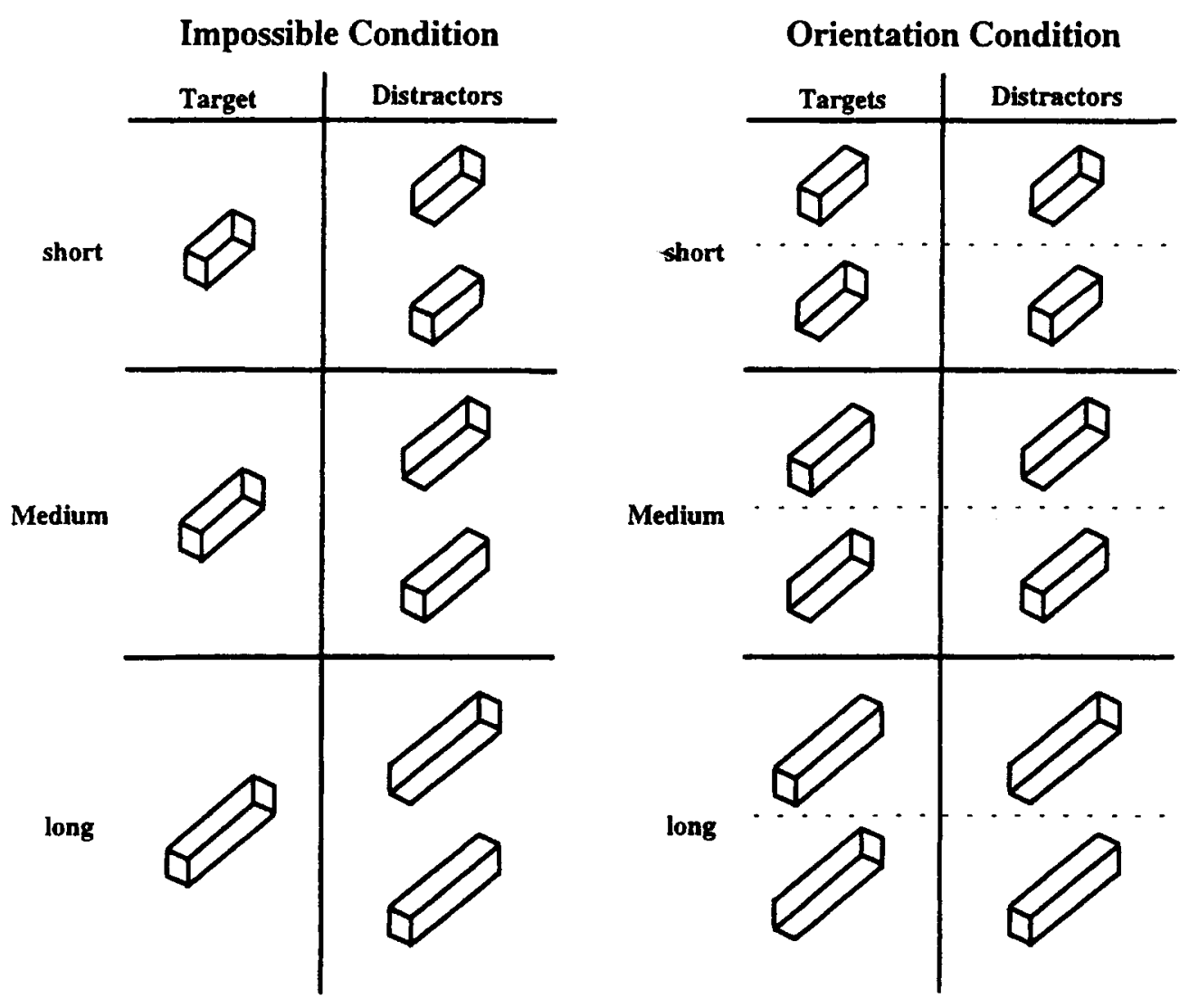

Figure 12. Target and distractor pairings used in the three length conditions (short, medium, and long) for both impossible (left) and orientation (right) target conditions of Experiment 5.

The results of Experiment 3 support the idea that impossible objects are inherently difficult to search for; however, there are three alternative ways of thinking about search for the block figure, such that our preferred interpretation of the results might be questioned. First, we have conceptualized the impossible block as having no global orientation. However, it might be that the two different orientations are treated separately in the sense that each block is broken down into two legal components. Considered in this way, the impossible block is a conjunction of two orientations. Second, a potentially important methodological consideration in explaining the results of Experiment 3 is that the distractors were heterogeneous (see Duncan \& Humphreys, 1989) and contained vertices, which, taken as a set, specify both component orientations. Therefore, it might be that the effortful search for the block target is a product of the nature of the heterogeneous distractors. Third, although the oriented impossible block was similar to the block figures used by Enns and Rensink (1991), we have not actually shown that its orientation can be detected in parallel. Therefore, it is conceivable that both possible and impossible versions of the block figure are difficult to process and that the results say nothing about the perceptibility of the impossible block itself. In sum, the findings of Experiment 3, though suggestive that im- possibility is inherently difficult to detect, are open to alternative interpretations. In Experiments 4 and 5, we sought clearer evidence that impossibility is not detected from the same representations that enable decisions about orientation to be made.

\section{EXPERIMENT 4}

In Experiment 4, we addressed these competing explanations of the results by comparing search in three conditions. In Conditions $\mathrm{B}$ and $\mathrm{C}$ the target item was the impossible block shape used in Experiment 3. In Condition $B$, the distractors were a homogeneous set of either all upward-oriented or all downward-oriented "possible" blocks. The identity of distractors varied randomly from trial to trial; however, if the search for the target block was based on the orientation of component $Y$ junctions, then search should still be efficient given that the target would contain a unique $\mathrm{Y}$ junction. In Condition $\mathrm{C}$, as in Experiment 3 , distractors were a mixture of upward- and downward-oriented possible blocks. In Condition A, the participants searched for a differently oriented possible block. All three conditions are depicted in Figure 10.

The critical comparisons were between Conditions A and $\mathrm{B}$ and between Conditions $\mathrm{B}$ and $\mathrm{C}$. If Conditions $\mathrm{A}$ 
and $B$ are the same, then the impossible figure will have been broken down into its component orientations. If they are different, the impossible block will have been shown to not be broken down into its component orientations. Comparing Conditions $\mathrm{B}$ and $\mathrm{C}$ was expected to reveal the effect of distractor heterogeneity on search for the impossible target.

\section{Method}

Participants, Apparatus, and Procedure. The participants, apparatus, and procedure were as described in the General Method section. Note that 8 participants took part in Experiment 4: 6 participants performed a unique ordering of the three conditions, and 2 participants performed the conditions in opposing orders of difficulty on the basis of the mean RTs of the previous 6 participants. Conditions A and B consisted of 276 trials, and Condition C consisted of 270 trials.

Stimuli. Target and distractor items in the three conditions are shown in Figure 10. Displays consisted of 4, 9, or 16 items arranged as in "rough" square. The size of display items was as in Experiment 3 (displays containing 16 items subtended $5.85^{\circ} \times 6^{\circ}$ ).

\section{Results}

RTs, percent errors, and standard deviations from mean RTs were analyzed as in Experiment 1. For RTs, all three main effects were significant [condition, $F(2,14)=13.31$, $p<.001$; response, $F(1,7)=26.89, p<.01$; set size, $F(2,14)=44.34, p<.0001$; see Figure 11]. None of the interactions were significant. Overall, "absent" responses were longer than "present" responses, and RTs increased linearly with set size. The main effect of condition was due to the longer RTs in Condition C. Present slopes in Conditions $\mathrm{A}, \mathrm{B}$, and $\mathrm{C}$ were 23,25 , and $47 \mathrm{msec} / \mathrm{item}$, respectively (absent slopes were 36,50 , and $68 \mathrm{msec} / \mathrm{item}$, respectively). Present RTs were clearly longer in Condition C. Conditions A and B were similar with respect to "present" responses; however, "absent" responses were longer in Condition B than in Condition A.

For errors, the main effects of response $[F(1,7)=32.59$, $p<.001]$ and set size $[F(2,14)=8.63, p<.01]$ were significant. In addition, the interactions between condition and set size $[F(4,28)=3.63, p<.05]$ and response and set size $[F(2,14)=14.47, p<.001]$ were significant. The participants made more errors on present trials, with misses increasing with set size. Errors increased more with set size in Conditions $\mathrm{C}$ and $\mathrm{A}$ than in Condition B.

Analysis of the standard deviations revealed no significant effects involving response and set size.

\section{Discussion}

The results for Conditions A and B were basically similar. The fact that the impossible block target was treated identically to the possible block is important. It implies that search is conducted on the basis of 3-D orientation as a property of local vertices, as opposed to an object property. Consequently, provided the target has at least one unique vertex orientation, it can be searched for relatively efficiently. As in Experiments 1 and 2, the rate and manner in which oriented targets were detected did not readily concord with those from other studies (e.g., Enns
\& Rensink, 1991). Although in Experiment 1 we argued for structural differences as being the cause of the apparently slow rate of orientation detection, the same is unlikely to have been true in Experiment 4. Here, it is possible that the variable mapping of orientation to targets and distractors across trials was important, though it is not clear why this should be the case. Importantly, our control experiments have removed stimulus quality and potential confounds with acuity as being the cause of the relatively slow orientation detections.

The conclusion concerning orientation agrees with that from Experiment 2. It implies that if we are to force participants to encode the impossible block, we must employ heterogeneous distractors. However, the comparison of Conditions B and C (and previous research) shows that searching among heterogeneous distractors will inevitably lead to a serial searching strategy. If we are to find conclusive evidence that impossibility itself requires an attentive process to detect it, we must find evidence that its detection interacts with some process known to operate in serial. We attempted to find such evidence in Experiment 5.

\section{EXPERIMENT 5}

In Experiment 5, we attempted to show that it is not simply distractor heterogeneity that is making search for the impossible object effortful. Work by Enns and King

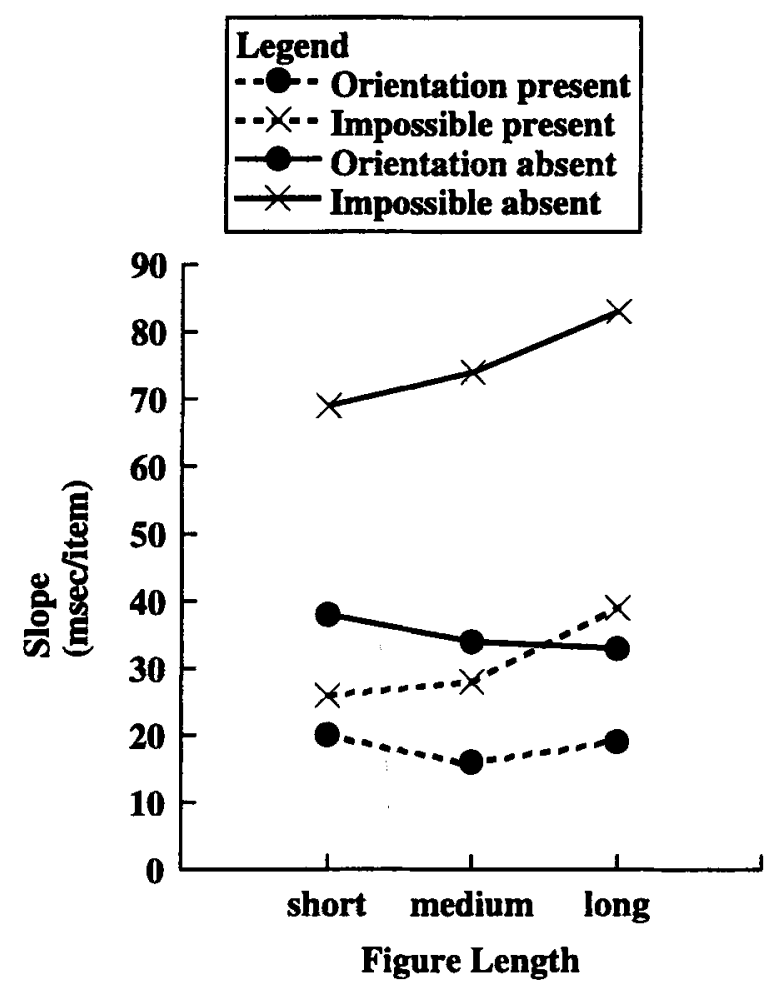

Figure 13. RT by set size slope values for target-present and target-absent trials for both impossible and orientation targets in Experiment 5 according to stimulus length (short, medium, and long). 

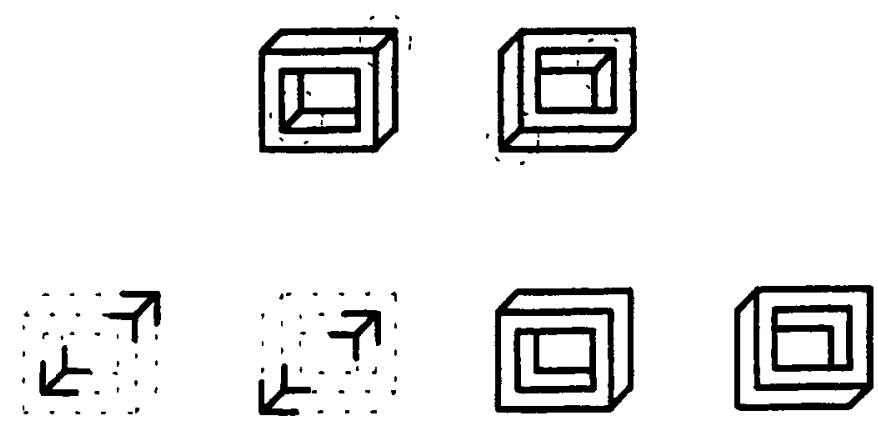

Figure 14. Downward (top left) and upward (top right) 3-D oriented box shapes employed in the present experiments. Both oriented boxes contain two line contours that terminate orthogonally at an arrow junction at one end and a $Y$ junction at the other, as highlighted bottom left. According to Enns and Rensink (1991), both of these junctions have a special role in determining 3-D orientation; however, generalty, our own control experiments indicate the external $\uparrow$ junctions to be critical in these particular stimuli.

(1990) and Cowie and Perrott (1993) suggests that consistency checking becomes less efficient as the distance between vertices increases. In Experiment 5, we investigated whether stimulus length would affect search for the impossible block target by varying the length of items. As a control, the same length manipulation was performed on block stimuli in which the target item differed from distractors in terms of 3-D orientation. The three length conditions for both orientation and impossible target items are depicted in Figure 12.

\section{Method}

Participants and Apparatus. The participants and apparatus were as described in the General Method section.

Stimuli. The six conditions (three involving search for an impossible target and three involving search for an orientation target) are shown in Figure 12. The six conditions consisted of three different lengths of items (short, medium, and long) for both orientation and impossible targets. Displays consisted of 4, 9, or 16 items, arranged as in Experiment 3. The size and position of display items were as in the previous experiments. The positions of the centers of display items were the same in all conditions.

Procedure. The procedure was as described in the General Method section. Each of the orientation conditions consisted of 276 trials; each of the impossible conditions consisted of 270 trials. Half of the participants performed the three orientation search conditions first; the other half performed the impossible conditions first. All participants performed the three length conditions.

\section{Results}

Mean RTs, percent errors, and standard deviations from mean RTs were subjected to 2 (condition: impossible and orientation) $\times 3$ (length: short, medium, and long) $\times 2$ (response: "present" and "absent") $\times 3$ (set size: 4,9 , and 16) ANOVAs. For RTs, the main effect of condition was significant $[F(1,5)=67.91, p<.01]$ with $\mathrm{RTs}$ longer to impossible targets. The main effects of length $[F(2,10)=$ $4.16, p<.05]$, response $[F(1,5)=42.68, p<.01]$ and set size $[F(2,10)=118.38, p<.01]$ were also significant, with absent RTs longer than present RTs. RTs increased as item length and set size increased. The interaction between condition and length was significant $[F(2,10)=$ $6.59, p<.05]$, as was the three-way interaction between condition, length, and set size $[F(4,20)=3.49, p<.05]$. Increasing the length of items had a significantly more deleterious effect on search for the impossible targets than on search for the 3-D orientation targets. This is illustrated in Figure 13, which graphs the present and absent RTs by set size slope values for both impossible and orientation targets according to stimulus length. The present search slopes in the orientation condition remained the same $(\approx 20 \mathrm{msec} / \mathrm{item})$ irrespective of stimulus length; in contrast, present and absent slopes increased with the length of the impossible target. Analysis of the standard deviations demonstrated no significant interactions involving both set size and response.

\section{Discussion}

Experiment 5 demonstrated that search for an impossible block target is affected by the length of stimulus. Search was significantly less efficient when display items were longer; however, the effect on search was not linearly related to stimulus length. Slope values for short and medium impossible targets were relatively similar ( 25 and $28 \mathrm{msec} / \mathrm{item}$, respectively), whereas the target-present slope for long targets was much greater ( $39 \mathrm{msec} / \mathrm{item})$. This suggests that the computation of impossibility is reliant on a consistency checking mechanism whose speed of operation to completion is scaled by distance. In other words, the difficulty of searching for the impossible figure in Experiments 3 and 4 was unlikely to have been solely a function of distractor heterogeneity. Furthermore, the fact that the distance effect was not just a main effect but interacted with display size suggests that each item was tested for the presence of two Y junctions. In contrast, judgments of orientation were not affected by distance. 
2D

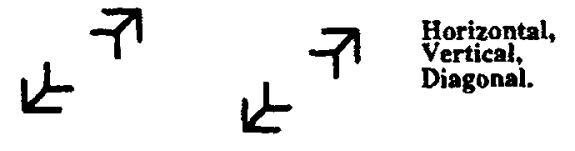

$3 \mathrm{D}$
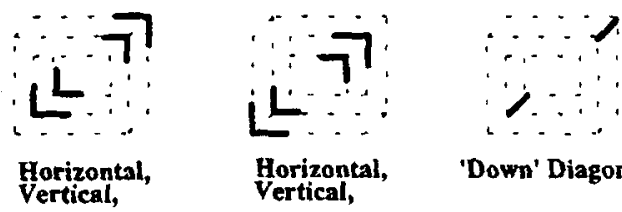

'Down' Diagonal
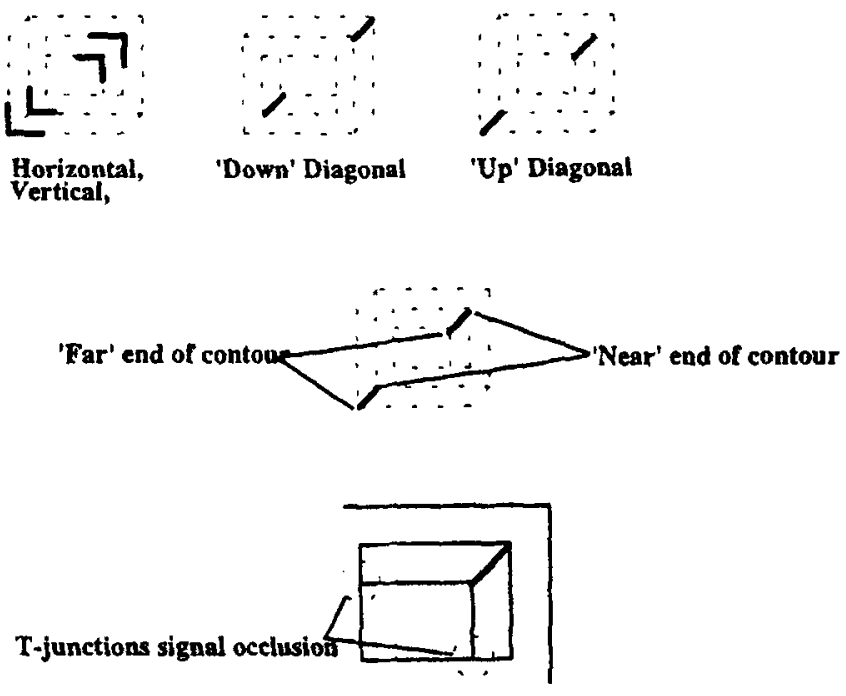

Figure 15. The contours and associated junctions predicted to be responsible for the 3-D orientation differences in the box stimuli illustrated in this figure consist of line elements with horizontal, ver(ical, and diagonal 2-D orientations (top). When the horizontal and vertical line contours are presented within a 3-D context (middle left), their orientation remains unchanged; however, when the diagonal line elements are presented in a 3-D context, their perceived orientation is altered (middle right). The two ends of the diagonal contours are perceived to be at different distances, "near" and "far." Presumably, in the case of the top diagonal, this interpretation is based on the occlusion of the horizontal and vertical contours, which are attached to the far end of the diagonal contour (bottom).

This finding is in line with those of Experiments 1,2, and 4 , in that orientation judgments made from a vertex will not be affected by the distance between two vertices.

\section{GENERAL DISCUSSION}

The aims of the present experiments were twofold: (1) to investigate the detection and perception of impossible figures, and (2) to examine how decisions about orientation were affected by possibility/impossibility. These two issues are linked by the status of object representations at the point when orientation information is available. The issues are not easy to investigate because of potential confounds, such as spatial resolution and distractor heterogeneity, but we have found some clear-cut results.

Neither of the impossible figures we tested (the box or block figure) could be detected preattentively. In Experiment 1 , when the participants were presented with the box figure, present search slopes were more than three times longer in search for impossibility than in search for a 3-D orientation difference of the same shape $(110 \mathrm{msec} /$ item vs. $30 \mathrm{msec} /$ item). Similar results were found with the block figure in Experiments 3 and 4.

The results of Experiment 5 indicate that a consistency checking procedure operates serially to detect impossibility. These results argue against the idea of an incoherence detection mechanism that could be used to direct attention (either overtly or covertly) to the site of the ambiguity to rectify the incoherence. Taken together, the results of Experiments 1, 3, 4, and 5 strongly suggest that incoherence/impossibility is not detected in early vision. These results suggest that the descriptions of objects computed in early vision must be incomplete.

In contrast, our results on the detection of orientation indicate that, although orientation was more difficult to detect than in some other studies (e.g., Enns \& Rensink, 1991), it is certainly easier to detect than impossibility. Of particular interest here is that our data from Experiment 1 were obtained using the box figure, which is more complex than the block figure typically used by Enns and Rensink. Nevertheless, the control experiments reported in the Discussion section of Experiment 2 show 
that the same issues of orthogonality and 3-D form are critical in determining the pattern of search. As discussed so far, the data are important in that they confirm our intuitions on impossibility detection and extend previous findings on orientation detection.

The fact that impossibility is detected extremely slowly (and certainly via a serial self-terminating search) whereas orientation can be detected faster and more efficiently suggests that decisions are not made from representations that are equally rich. One way of explaining the ability of the participants to search for 3-D orientation, while being unable to search for impossibility, is to suggest that 3-D orientation is a local property compared with impossibility, which is an idiosyncratic feature (i.e., a particular $T$ junction) of the global set of line relations (i.e., shape). To investigate this issue, in Experiment 2, we employed impossible shapes as display items while the participants searched for a 3-D orientation target. If 3-D orientation is a property assigned to a coherent set of line labels (i.e., a possible object), then we would expect search for 3-D orientation to be impeded if no coherent set of line labels could be applied to a given shape (i.e., an impossible object). The three conditions of Experiment 2 compared search for a 3-D orientation target when shapes were possible or impossible. The results demonstrated that search for 3-D orientation among possible and impossible shapes did not differ a great deal. Present slope values were 31 and $36 \mathrm{msec} / \mathrm{item}$ for possible and impossible display items, respectively. This suggests that the participants did not generate, for each display item, a coherent set of line labels to which a 3-D orientation was assigned, since this would not be feasible for the impossible shapes.

The inability to generate a globally coherent set of labels for the impossible shapes ensures that participants are likely to derive 3-D orientation locally. Figure 14 illustrates that both the upward-oriented and the downwardoriented box shapes used in Experiment 2 possess the same orientation-defining junctions (see Experiment 2 of Enns \& Rensink, 1991). Thus, the differently oriented items do not appear to be differentiated by the presence/ absence of a single junction of a particular orientation. How, then, do participants differentiate between the two differently oriented display items? It could be reasoned that 3-D orientation is not a property of a junction per se but is assigned to a contour on the basis of junction information. The perceived orientation of the horizontal and vertical line elements from which the junctions in the bottom left of Figure 14 are composed is not altered when they are presented within a 3-D context. In contrast, Figure 15 illustrates that the perceived orientation of the diagonal lines is altered when presented within a 3-D context. In other words, the orientation of these lines, in upward- and downward-oriented context, is not specified by a particular junction presented at one end of the contour. Instead, perceived orientation must be based on the pattern of relations of the horizontal and vertical contours with respect to the horizontal and vertical junction com- ponents. Together, these specify which end of the diagonal contour is nearest in 3-D space.

This suggests that a great deal of processing of the relations between lines is required to establish the 3-D orientation of the shapes employed in Experiment 2. However, if this were the case, then we might expect the incoherence present in the impossible display items to impede orientation search as they are disrupting the occlusion relations required to assign relative depths to the two ends of the diagonal line contour. One straightforward explanation of why search is not impeded greatly is that the inconsistency in the impossible box shape only affects the assignment of 3-D orientation to a subset of the internal diagonal contours, whereas 3-D orientation might be derived only for the diagonal contours that are part of the outline of the shape. As the results of the control conditions in Table 1 suggest, this account seems plausible.

Local coding for orientation also seems to account for the results from Experiment 4 . The impossible version of the block shape incorporates the two "ends" of its possible equivalents, which were either upward- or downwardoriented blocks. It was reasoned that if 3-D orientation was coded as a property of an object, then either the impossible block should have no global orientation due to its two incompatible orientations (which we know not to be the case from Experiment 2) or it should be randomly assigned either an upward or downward orientation depending on which $\mathrm{Y}$ junction was used to assign the impossible blocks global orientation (global account). If 3-D orientation essentially reduces to simply possessing a particular $\mathrm{Y}$ junction, then the impossible block shape will, in effect, have two orientations (since it has two $\mathrm{Y}$ junctions) (local account). Both accounts predict that Condition $\mathrm{C}$, in which the impossible block was presented among both upward and downward possible blocks, would be difficult because the impossible target would share its orientation(s) with either some (global account) or all (local junction account) of the distractor items. However, the two accounts would predict different patterns of performance in Conditions A and B.

According to the global account, the impossible target in Condition B would, on a subset of trials, share its orientation with the distractor items, making it difficult to search for, relative to Condition $A$ in which the target is always uniquely specified in terms of orientation. In contrast, the local junction account would predict that search performance would be the same in the two conditions, since the target always has a unique orientation-defining $Y$ junction. The performance of the participants in Conditions A and B was similar, consistent with 3-D orientation being a local property of junction labels. Thus, in Condition B, the participants were able to detect the target by the presence of an "odd-one-out" Y junction. When this target did not possess a unique $Y$ junction, then search resorted to a laborious checking of display items for an item with two "incompatible ends," a process that appears to be spatially limited (Experiment 5). 
In conclusion, the difficulty that participants encounter searching for impossible target objects demonstrates that line relations are not processed fully in early vision, unlike some computer-based line-labeling algorithms. Instead, local "clusters" of lines appear to be processed sufficiently to allow the derivation of 3-D orientation, but not the detection of global incoherence.

\section{REFERENCES}

Ballard, D. H., \& Brown, C. M. (1982). Computer vision. Englewood Cliffs, NJ: Prentice-Hall.

Biederman, I., \& Ju, G. (1988). Surface versus edge-based determinants of visual recognition. Cognitive Psychology, 20, 38-64.

CoWIE, R., \& PERrotT, R. (1993). From line drawings to impressions of 3-D objects: Developing a model to account for the shapes that people see. Image \& Vision Computing, 11, 342-352.

DAvidoff, J. (1991). Cognition through color. Cambridge, MA: MIT Press.

Donnelly, N., Weekes, B. S., Humphreys, G. W., \& Albon, A. (1998). Processes involved in the computation of a shape description. Journal of Experimental Psychology: Human Perception \& Performance, 24, 1119-1130.

DunCan, J., \& Humphreys, G. W. (1989). Visual search and stimulus similarity. Psychological Review, 96, 433-458.

ENNS, J. T., \& KING, K. A. (1990). Components of line-drawing interpretation: A developmental study. Developmental Psychology, 26, 469-479.

EnNs, J. T., \& Rensink, R. A. (1991). Pre-attentive recovery of three- dimensional orientation from line drawings. Psychological Review, 98, 335-351.

Humphreys, G. W., \& Donnelly, N. (1998). 3-D constraints on spatially parallel shape perception. Manuscript submitted for publication. Humphreys, G. W., Keulers, N., \& Donnelly, N. (1994). Parallel visual coding in three-dimensions. Perception, 23, 453-470.

LofTUS, G. R., \& Masson, M. J. (1994). Using confidence intervals in within-subject designs. Psychonomic Bulletin \& Review, 1, 476-490.

OstergaARD, A. L., \& Davidoff, J. B. (1985). Some effects of color on naming and recognition of objects. Journal of Experimental Psychology: Learning, Memory, \& Cognition, 11, 579-587.

SugihaRA, K. (1982). Classification of impossible objects. Perception, 11, 65-74.

TownsEND, J. T. (1972). Some results on the identifiability of parallel and serial processes. British Journal of Mathematical \& Statistical Psychology, 25, 168-199.

Treisman, A., \& Gelade, G. (1980). A feature-integration theory of attention. Cognitive Psychology, 12, 97-136.

WALTZ, D. (1975). Understanding line drawings of scenes with shadows. In P. H. Winston (Ed.), The psychology of computer vision (pp. 19-91). New York: McGraw-Hill.

Wolfe, J. M. (1994). Guided Search 2.0: A revised model of visual search. Psychonomic Bulletin \& Review, 1, 202-238.

WoLFE, J. M. (1997). What can 1 million trials tell us about visual search. Psychological Science, 9, 33-39.

(Manuscript received January 31, 1997;

revision accepted for publication April 24, 1998.) 